\title{
Trichoscopic Findings of Frontal Fibrosing Alopecia
}

A.M.Farag, R.M.Salem; A.M.Abdelrahman and D.M.EL-Adawy

Dermatology \& Andrology. Dept., Faculty of Medicine, Benha Univ., Benha, Egypt

E-Mail:Dina2@gmail.com

\begin{abstract}
:
Frontal Fibrosing Alopecia (FFA) is a type of scarring alopecia known as a clinical variant of lichen planopilaris (LPP). It is characterized by slowly progressive scarring alopecia on the hairline and affects explicitly postmenopausal women. The point of the current examination was to survey trichoscopic discoveries in instances of Frontal Fibrosing Alopecia. The examination was led on 50 female patients with frontal fibrosing alopecia who were selected from Dermatology outpatient center of Benha University clinic in the period from January 2019 to January 2020. A composed educated assent was acquired from all members. The examination was endorsed by the nearby morals board on look into including human subjects of Benha Faculty of Medicine. All patients were exposed to Dermatological assessment for clinical appraisal and trichoscopic assessment of frontal fibrosing alopecia. Trichoscopy featured a checked decrease in the quantity of follicular ostia, perifollicular desquamation, perifollicular blue-dim spots Follicular hyperkeratosis, Perifollicular erythema, Lonely hair, unexpected interference of the hairline, with the nonattendance of the vellus hairs that are regularly seen in typical scalp. Trichoscopy is a useful apparatus in the conclusion of frontal fibrosing alopecia.
\end{abstract}

\section{Introduction}

Frontal Fibrosing Alopecia (FFA) is a kind of scarring alopecia known as a clinical variation of lichen planopilaris (LPP) [1]. It is described by gradually dynamic scarring alopecia on the hairline and influences expressly postmenopausal ladies. A seriousness score was proposed to be viably utilized for classifying FFA patients in clinical practice and in look into contemplates [2].

Clinically, FFA presents for the most part as a band-like downturn of the frontotemporal hairline. The alopecic skin is somewhat atrophic, without follicular ostia, smooth, and lighter than the constantly sununcovered temple skin [3].

Three clinical examples of male pattern baldness have been portrayed in FFA, as per the various sorts of hairline downturn depicted throughout the years: straight, diffuse crisscross, and pseudo-periphery. The straight example is the band of uniform frontal hairline downturn without loss of hair thickness behind the hairline. The diffuse crisscross example is equivalent to straight however with in any event half diminished hair thickness [4].

Pseudo-periphery hairline downturn is a clinical introduction like footing alopecia (subsequently the term 'pseudo') where the periphery sign is the nearness of some hair held along the hairline (particularly in the fleeting region) in front of the alopecic skin. Throughout the years, instances of separated occipital or retro-auricular or sideburns FFA have been accounted for, building up that this ailment isn't kept to the frontal scalp [5].

Sidelong or complete eyebrow misfortune, infrequently with perifollicular and entomb follicular erythema, is an extremely regular component of FFA [2]. Volume misfortune has been accounted for the eyelashes. Diminishing of axillary, pubic, appendage, and truncal hair, some of the time related with follicular keratosis as well as erythema, can likewise happen previously or after the scalp male pattern baldness. These highlights are normally mistaken for age-related body male pattern baldness and never revealed by patients themselves [6].
Great lichen planus in other scalp zones or other body territories just as lichen planus pigmentosus and less regularly sorrow of the facial veins have likewise been depicted in FFA-influenced patients [7]. Facial erythema, at times connected with follicular keratosis, might be diffuse or confined in the temple and present as red dabs [8]. Follicular hyperkeratosis (peripilar throws) and perifollicular erythema are seen [8].

\section{Patients and method}

The study was approved by the local ethics committee on research involving human subjects of Benha Faculty of Medicine. A written informed consent was obtained from all participants. The study was conducted on 50 female patients of frontal fibrosing alopecia were recruited from Dermatology outpatient clinic of Benha University hospital in the period from January 2019 to January 2020.

\section{All patients were subjected to the following \\ r.l Full history taking}

- Personal history: name, age, special habits of medical importance.

- Present history: age of onset, duration of disease

- Past history (Hypertension, drugs, diabetes mellitus, thyroid disease, lichen planus, rheumatoid arthritis, systemic lupus)

\section{Family history of FFA}

\subsection{Clinical Examination}

\subsection{General examination}

- Each subject's weight in kilograms and height in meters was measured

- $\quad$ BMI The formula is BMI $=\mathrm{kg} / \mathrm{m}^{2}$ where $\mathrm{kg}$ is a person's weight in kilograms and $\mathrm{m}^{2}$ is their height in meters squared.) A BMI of 25.0 or more is overweight, while the healthy range is 18.5 to 24.9 . BMI applies to most adults 18-65 years [9].

\section{Local and skin examination}

- $\quad$ Skin was examined for other skin diseases 
- Dermatological examination for clinical assessment of frontal fibrosing alopecia.

- Trichoscopic examination.

\section{Results}

Table (1) History findings in the studied cases.
The study included 50 female patients with frontal fibrosing alopecia. Their age ranged from 39 to 60 years old. The age of disease onset ranged from 38 to 58 years old. The disease duration ranged from 1 to 3 years Table (1).

\begin{tabular}{lll}
\hline & & Patients $\mathbf{N = 5 0}$ \\
\hline Patients age (years) & Range & $39: 60$ \\
Age of onset (years) & Range & $38: 58$ \\
Duration (years) & Range & $1: 3$ \\
\hline
\end{tabular}

$\mathrm{SD}$, standard deviation.

The disease was associated with comorbidities in most of the patients Fig (1).

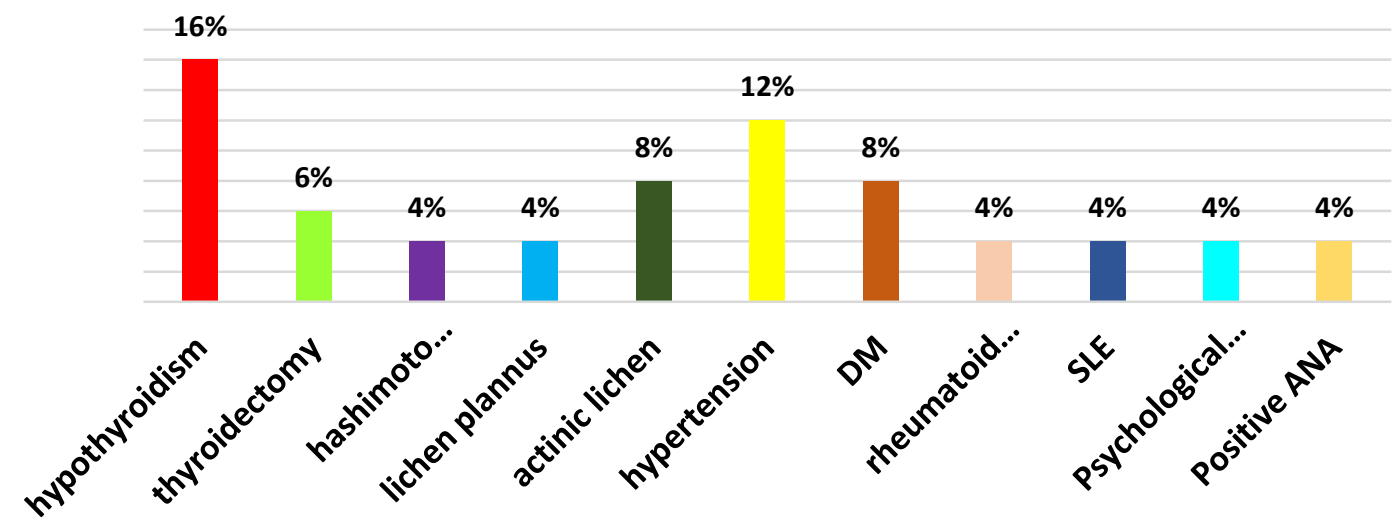

Fig (1) Comorbidities in all studied cases.

Clinical examination revealed the following clinical signs: frontotemporal and frontoparietal hair recession, eyebrows and eyelashes loss, facial papules and depressed facial viens Fig $(2,3)$.

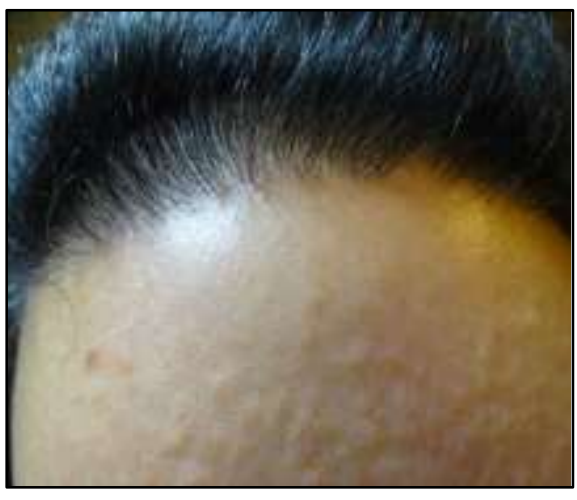

Fig (2) FFA with prominent facial papules.

\section{Trichoscopic findings}

Trichoscopy highlighted a marked reduction in the number of follicular ostia, perifollicular desquamation, perifollicular blue-gray dots Follicular hyperkeratosis,

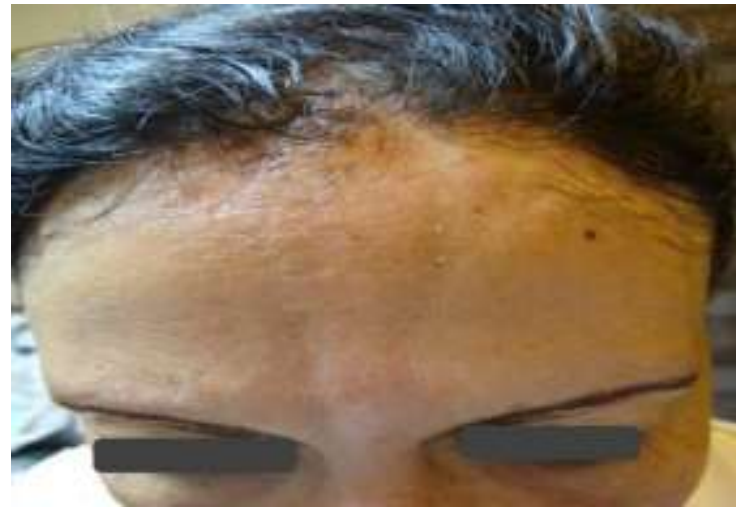

Fig (3) FFA; Anterior hair line recession, eyebrows affection, depressed facial veins.

Perifollicular erythema, Lonely hair , abrupt interruption of the hairline, with the absence of the vellus hairs that are typically observed in normal scalp Fig (4). 


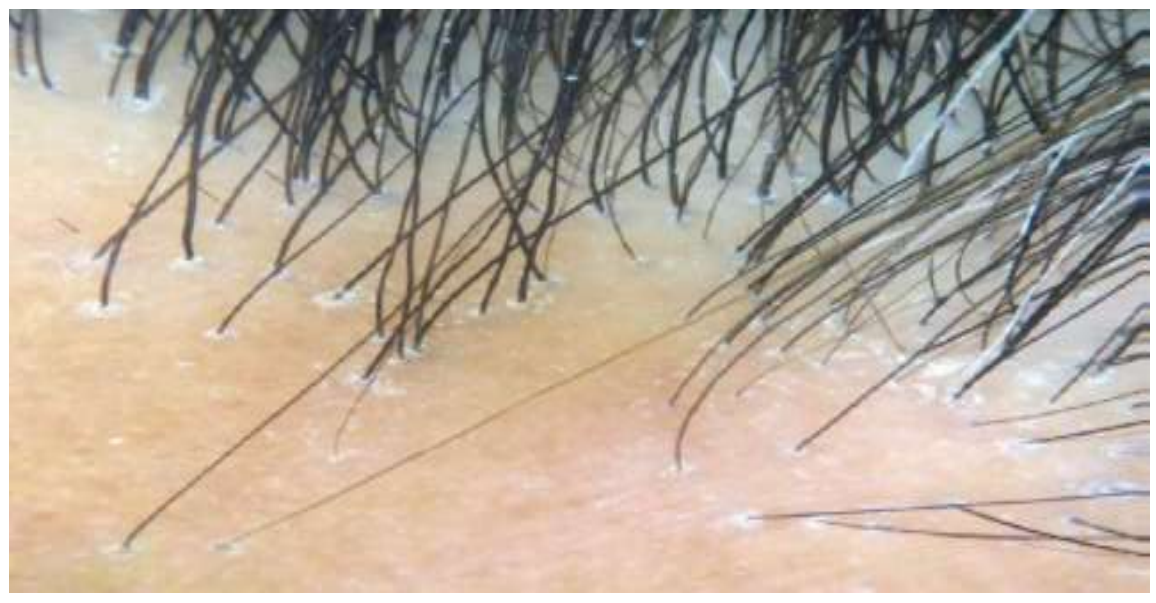

Fig (4)Trichoscopy shows loss of vellus hairs, loss of follicular opening, perifollicular scaling, inter-follicular scales and lonely hairs.

\section{Discussion}

Related comorbidities in the present cases included hypothyroidism, had thyroidectomy, Hashimoto thyroiditis, Lichen planus, actinic lichen, hypertension, Diabetes Mellitus, Rheumatoid joint pain A, SLE, mental issues, and positive ANA.

An observational, cross-sectional and distinct investigation was directed in France and Germany (in the Center de Sant_e Sabouraud, Paris, France, and the Department of Dermatology and Allergy of the Charit_e - Universit€atsmedizin Berlin, Germany. Between August 2013 andApril 2018,) found that of the most generally detailed comorbidities incorporate thyroid capacity issue (38\% of ladies), blood vessel hypertension (18\% of women)and lipid metabolic disarranges (22\% of ladies ) [10].

Conveyance of male pattern baldness contrasted between contemplated subjects; frontoparietal, and frontotempoal, eye forehead misfortune and eye lashes misfortune. High hairline is a basic finding for frontal fibrosing alopecia conclusion. Fronto-parieto-transient hairline downturn for the most part grows reciprocally and symmetrically] and it can reach out through to the retro auricular locale and occipital edge Additionally, frontal fibrosing alopecia may cause torment, tingling or consuming sensations in the band over the frontal hairline. Two-sided eyebrow misfortune is another clinical admonition sign for frontal fibrosing alopecia, and may speak to perhaps the most punctual appearance which could go before alopecia by a while to years. The distal third is ordinarily influenced and, in contrast to the scalp, it is bizarre to discover clear erythema or scaling on the eyebrow zone. Eyelash misfortune may likewise happen, More clear frontal veins can be distinguished by palpation as a restricted misery, close to the first hairline. Cutaneous decay is believed to be mindful. Facial papules in frontal fibrosing alopecia were portrayed as follicular, normochromic and monomorphic papules, haphazardly dispersed on facial skin and are not handily seen, being better envisioned over the sanctuaries [11].
Trichoscopic discoveries incorporate the nonappearance of follicular openings and vellus hair in the frontal hairline, follicular hyperkeratosis, perifollicular scaling, and erythema, perifolicular scaling is commonly less successive and milder in FFA than LPP. However, pili torti was noted all the more much of the time uncovering an accommodating marker for FFA. In addition, as a novel finding an examination showed that vellus hairs can be obvious in FFA [12].

In the present examination Trichoscopy featured a checked decrease in the quantity of follicular ostia, perifollicular desquamation, perifollicular blue-dark spots Follicular hyperkeratosis, Perifollicular erythema, Lonely hair , unexpected interference of the hairline, with the nonattendance of the vellus hairs that are regularly seen in typical scalp

FFA might be considered as a trichological crisis which needs appropriate finding and early treatment to stop the hair follicles fibrosis and protect the rest of the hairs.

\section{Refrences}

[1] S. Kossard, Postmenopausal frontal fibrosingalopecia. Scarring alopecia in a patterndistribution. Arch Dermatol, Vol.130, pp.770-774,1994.

[2] D. Saceda-Corralo, O.M. Moreno-Arrones, P. Fonda-Pascual, Development and validation of the frontal fibrosing alopecia severity score. J Am Acad Dermatol, Vol.78, pp.522-9,2018.

[3] P. Fonda-Pascual, D. Saceda-Corralo, O.M. Moreno-Arrones, Frontal fibrosing alopecia and environment: may tobacco be protective? Defining environmental impact on FFA patientsand a possible protective influx of smoking habit. J Eur Acad Dermatol Venereol, Vol.31, pp.e98-9,2017.

[4] P. Mirmirani, B. Zimmerman, Cocking the eyebrows to find the missing hairline in frontal fibrosing alopecia: a useful clinical maneuver. J Am Acad Dermatol, Vol.75, pp.e63,2016. 
[5] O.M. Moreno-Arrones, D. Saceda-Corralo, P. Fonda-Pascual, Frontal fibrosing alopecia: clinical and prognostic classification. J Eur Acad Dermatol Venereol, Vol.31, pp.1739-45,2017.

[6] A.A. Diaz, M. Miteva Peripilar, "guttate" hypopigmentation of the scalp and idiopathic guttate hypomelanosis in frontal fibrosing alopecia. Skin Appendage Disord, Vol.50, pp.130-45,2018

[7] V. Meyer, M. Sachse, C. Rose, Follicular red dots of the hip in frontal fibrosing alopecia-do we have to look twice? J Dtsch Dermatol Ges, Vol.15, pp.327-8,2017.

[8] A.C. Katoulis, K. Diamati, D. Sgouros, Frontal fibrosing alopecia and vitiligo: coexistence or true association? Skin Appendage Disord, Vol.2, pp.152-52,017.

[9] Trefethen, Nick, "Institute, University of Oxford. Retrieved 5 February, Vol.25, pp.140-30,2019

[10]A. Alegre-Sanchez, D. Saceda-Corralo, C. Bernardez, ,Frontal fibrosing alopecia in malepatients: a report of 12 cases. J Eur Acad Dermatol Venereol, Vol.31,pp. e112-e14,2017.

[11]L. Esteban-Lucía, A.M. Molina-Ruiz, L. Requena. Update on frontal fibrosing alopecia . Actas Dermosifiliogr, Vol. 108 ( 4 ), pp.293 - 304 ,2017.

[12]R. Abedini, K. Kamyab Hesari, M. Daneshpazhooh, Validity of trichoscopy in the diagnosis of primary cicatricialalopecias.Int Dermatol, Vol.55, pp.1106-14,2016 